\title{
Detección de enterobacterias productoras de $\beta$-lactamasas de espectro extendido (BLEE) aisladas en carne molida de supermercados de un distrito de Lima, Perú
}

\author{
Detection of Extended-spectrum $\beta$-lactamases (ESBL)-producing Enterobacteriaceae isolated from ground \\ beef in supermarkets of a district of Lima, Peru
}

Valeria Cortez Sandoval ${ }^{1}$, Carlos Shiva Ramayoni ${ }^{1}$

\section{RESUMEN}

El objetivo del estudio fue detectar la presencia de enterobacterias productoras de $\beta$-lactamasas de espectro extendido (BLEE), aisladas de carne molida, proveniente de recortes de res que se comercializan en supermercados de un distrito de Lima, Perú. Se recolectaron muestras de 50 gramos de carne molida de 10 tiendas de 5 cadenas de supermercados, las que se procesaron en el Laboratorio de Nutrición e Inocuidad Alimentaria de la Facultad de Medicina Veterinaria y Zootecnia de la Universidad Peruana Cayetano Heredia. Se realizaron diluciones para cada muestra y se sembraron en Agar EMB. A través de baterías de bioquímica comercial se identificaron las especies de enterobacterias. Se utilizaron dos métodos para evidenciar el patrón BLEE, el primero para determinar cepas sospechosas (método de difusión simple en agar) y el segundo para confirmar la producción de BLEE (método de doble disco). Se aislaron 38 cepas de enterobacterias, resultando el 28,94\% (11) sospechosas de producir BLEE para los antibióticos cefotaxima, ceftazidima, ceftriaxona, aztreonam y cefpodoxima bajo el método de difusión simple. Resultando positivas a BLEE por método de doble disco el 15,79\% (6) a los mismos antibióticos más amoxicilina con ácido clavulánico. Se pone en evidencia que la carne molida comercializada en supermercados puede contener cepas productoras de BLEE, siendo las especies de enterobacterias más frecuentes Citrobacter freundii, Serratia marcescens, Yersinia enterocolitica, Citrobacter braakii y Enterobacter cloacae.

PALABRAS CLAVE: entereobacterias, BLEE, carne molida, resistencia antibiótica.

\section{SUMMARY}

The aim of the study was to detect the presence of Extended-spectrum $\beta$-lactamases (ESBL)- producing Enterobacteriaceae isolated from ground beef in supermarkets of a district of Lima, Peru. Samples of 50 grams of ground beef were collected from 10 stores of 5 supermarkets. Samples were processed in the Laboratorio de Nutrición e Inocuidad Alimentaria de la Facultad de Medicina Veterinaria y Zootecnia de la Universidad Peruana Cayetano Heredia. Dilutions were made for each sample and placed in EMB agar. The enterobacteria species were identified by commercial biochemical batteries. Two methods were used to demonstrate the ESBL pattern, the first to determine the suspects strains (simple diffusion method in agar) and the second to confirm if they were producers of ESBL (double disc method). Thirthy eight strains of Enterobacteria were isolated, it was 28.94\% (11) of strains suspected of producing ESBL for the antibiotics cefotaxime, ceftazidime, ceftriaxone, aztreonam and cefpodoxime under the simple diffusion method. Resulting positive strains to ESBL by double disc method were $15.79 \%$ (6) to

\footnotetext{
Facultad de Medicina Veterinaria y Zootecnia, Universidad Peruana Cayetano Heredia. Lima, Perú.
} 
the same antibiotics and amoxicillin with clavulanic acid. Commercial ground beef of supermarkets may contain ESBL-producing strains, the most frequent Enterobacteria species were Citrobacter freundii, Serratia marcescens, Yersinia enterocolitica, Citrobacter braakii and Enterobacter cloacae.

PALABRAS CLAVE: Enterobacteriaceae, ESBL, ground beef, antimicrobial resistance

\section{INTRODUCCIÓN}

Las enterobacterias son uno de los agentes infecciosos más importantes, junto con el estafilococo dorado, reportados en infecciones nosocomiales debido a su capacidad de mutación y asociación genética, que permite desarrollar enzimas ( $\beta$-lactamasas de espectro extendido) que confieren cada vez mayor resistencia a los antibióticos. Esto representa un problema de salud pública por la dificultad en los tratamientos, sobre todo, hospitalarios (Díaz, Hernández, MartínezMartínez, Rodríguez-Baño y Pascual, 2009; Perozo y Castellano, 2009; García-Hernández et al., 2011).

Éstas bacterias se encuentran distribuidas de forma cosmopolita, incluyendo en los alimentos de origen animal como la carne de bovinos (Codex Alimentarius [CODEX], 2005). En la industria alimentaria, la calidad microbiológica es fundamental para asegurar la inocuidad de los alimentos a los consumidores; debido a que algunos microorganismos pueden encontrarse contaminando en distintos eslabones de la cadena productiva (CODEX, 2005; Jay, Loessner y Golden, 2005; Servicio Nacional de Seguridad Agraria. [SENASA], 2012). En el caso de enterobacterias productoras de $\beta$-lactamasas de espectro extendido (BLEE), la importancia radica en la capacidad de transferencia de genes de resistencia a través de plásmidos (codificación plasmídica) de una bacteria donadora a una receptora. Los genes de resistencia transmisibles conllevan a resistencias cruzadas frecuentes que se pueden adquirir contra antibióticos como aminoglucósidos, tetraciclinas, entre otros (Blanc, 2007; Díaz et al., 2009; GarcíaHernández et al., 2011).

Las BLEE son enzimas que conceden resistencia a todos los $\beta$-lactámicos, ejerciendo un efecto hidrolítico, principalmente en penicilinas y cefalosporinas de amplio espectro y monobactámicos. Por otro lado, no ejercen este efecto sobre carbapenémicos, cefamicinas, ni combinaciones de $\beta$-lactámicos con inhibidores de $\beta$-lactamasas tales como el ácido clavulánico, tazobactam y sulbactam. También existen $\beta$-lactamasas mediadas por derivados de otras enzimas con menor espectro hidrolítico, las llamadas $\beta$-lactamasas cromosómicas. Asimismo, cuando el mecanismo es la hiperproducción, se obtienen características similares a las $\beta$-lactamasas clásicas, mediadas por bacterias como Citrobacter diversus, Klebsiella oxytoca, Yersinia enterocolítica y especies del género Kluyvera; este último posee un nuevo tipo de BLEE de relevancia (CTX-M) (Oliver y Cantón, 2005; Seral, Pardos y Castillo, 2010; GarcíaHernández et al., 2011).

Hoy en día se conocen múltiples variedades de BLEE; las más importantes son las del tipo TEM-4, TEM-24, TEM-52, SHV-12, CTX-M-9, CTX-M-14, CTX-M-3, CTX-M-15 y CTXM-32. Los métodos de detección incluyen análisis moleculares y de sensibilidad microbiana a través de un antibiograma que permita detectar fenotipos compatibles (Díaz et al., 2009; Perozo y Castellano, 2009).

Debido a la amplia distribución de estos microorganismos (aguas, suelos, alimentos, plantas, heces), forman parte de un sistema biológico e integrado en donde intervienen animales de beneficio y el hombre, generando potencial daño sobre la salud pública. Adicionalmente, la capacidad de las BLEE de conceder resistencia a las enterobacterias favorece el desarrollo y perpetuación de las enfermedades.

El objetivo del presente estudio fue detectar enterobacterias productoras de BLEE aisladas, a partir de carne molida comercializada en supermercados de un distrito de Lima (Miraflores), Perú.

\section{MATERIAL Y MÉTODOS}

\section{Diseño del estudio y toma de muestras}

Se incluyó a todos los supermercados del distrito de Miraflores, Lima, Perú. La unidad muestral fue carne molida proveniente de cortes de res, exhibida en bandejas de poliestireno, cubierta con film de polietileno tereftalato de baja densidad y almacenada en expendio al público a temperatura de refrigeración $\left(4^{\circ} \mathrm{C}\right)$. Cada muestra estuvo conformada por 50 
gramos de carne molida expendidas en bolsas de plástico del mismo establecimiento. Se tomaron dos muestras por tienda de un total de diez tiendas de las cinco cadenas de supermercados presentes en el distrito de Miraflores entre marzo y abril del 2016. Las muestras fueron transportadas bajo cadena de frío $\left(4^{\circ} \mathrm{C}\right)$ a través de un contenedor termoaislante para su procesamiento microbiológico en el Laboratorio de Nutrición e Inocuidad Alimentaria de la Facultad de Medicina Veterinaria y Zootecnia de la Universidad Peruana Cayetano Heredia.

El procesamiento fue realizado dentro de las 2 primeras horas de recolección en el Laboratorio de Nutrición e Inocuidad Alimentaria de la Facultad de Medicina Veterinaria y Zootecnia de la Universidad Peruana Cayetano Heredia, bajo estrictas medidas de bioseguridad.

\section{Procesamiento microbiológico}

Se pesó 1 gramo de cada muestra de carne molida y se homogenizó en $9 \mathrm{ml}$ de agua destilada estéril (Maturin y Peeler, 2001). Se realizaron diluciones seriadas, se tomó $0,1 \mathrm{ml}$ y con un asa de Digralsky se sembró en Agar EMB (Becton Dickinson GmbH, 2013). Se incubó de 24 a 48 horas a $35 \pm 2^{\circ} \mathrm{C}$ en condiciones de aerobiosis. Luego se realizó el conteo de colonias y se multiplicó por el factor de dilución, obteniendo el valor de unidades formadoras de colonias (UFC), ajustando el resultado a 2 dígitos y potencias de 10. Cinco colonias por placa se tomaron seguidamente para realizar la identificación básica preliminar. Para la identificación definitiva se utilizó el kit comercial de bioquímica API 20E de BioMérieux (2017).

El análisis de susceptibilidad antimicrobiana se realizó en agar Mueller Hinton (Becton Dickinson $\mathrm{GmbH}$, 2017). Para garantizar la reproducibilidad se estandarizó el inóculo bacteriano a una escala 0.5 de Mac Farland, equivalente entre $1-2 \times 10^{8}$ UFC $/ \mathrm{ml}$ por espectrofotometría a $625 \mathrm{~nm}$ (Clinical Laboratory Standards Institute [CLSI], 2015).

Se hizo un primer antibiograma (método de difusión simple) para detectar las cepas de enterobacterias sospechosas de producir BLEE; seguidamente, se realizó un segundo antibiograma (método de doble disco) para la confirmación de producción de BLEE (Jarlier, Nicolas, Fournier y Phillippon, 1998). Se utilizó un grosor de agar de $4 \mathrm{~mm}(20 \mathrm{ml}$ aproximadamente, $\mathrm{pH} 7 \pm 0.1$.) sobre placas Petri estériles de $90 \mathrm{~mm}$ de diámetro. En ambos casos, los antibiogramas se incubaron durante 24 horas a $35 \pm$ $2^{\circ} \mathrm{C}$.

Los antibióticos utilizados para el método de difusión simple fueron cefotaxima (CTX) $30 \mu \mathrm{g}$, ceftazidima (CAZ) $30 \mu \mathrm{g}$, ceftriaxona (CRO) 30 $\mu \mathrm{g}$, aztreonam (ATM) $30 \mu \mathrm{g}$ y cefpodoxima (CPD) $10 \mu \mathrm{g}$, (Oxoid, Inglaterra). Para el método de doble disco se utilizaron los mismos antibióticos además de amoxicilina con ácido clavulánico (AMC) $30 \mu \mathrm{g}$ (Jarlier et al., 1998). Cabe mencionar que los discos fueron distribuidos a $24 \mathrm{~mm}$ de distancia del disco central que contenía AMC. Se determinó como cepa BLEE sospechosa aquellas que presentó un halo de inhibición $\leq 17 \mathrm{~mm}$ para cefpodoxima, $\leq 22 \mathrm{~mm}$ para ceftazidima, $\leq 27 \mathrm{~mm}$ para aztreonam, $\leq 27 \mathrm{~mm}$ para cefotaxima, $\leq 25 \mathrm{~mm}$ ceftriaxona (CLSI, 2015). Para el método de confirmación (método de doble disco), se determinó como cepa productora de BLEE a aquella que generó halo y distorsión entre AMC y los antibióticos circundantes, a una distancia de $24 \mathrm{~mm}$ (Jarlier et al., 1998; Perozo y Castellano, 2009).

Análisis de datos

Se utilizaron tablas de frecuencia para registrar la presencia de las cepas de enterobacterias y su

Tabla 1. Evidencia de enterobacterias en muestras de carne (UFC/g) aisladas de supermercados en el Distrito de Miraflores, Lima - Perú. Año 2016.

\begin{tabular}{lcc}
\hline & \multicolumn{2}{c}{ Enterobacterias } \\
\cline { 2 - 3 } Lugar de muestreo & Muestra 1 & Muestra 2 \\
\hline Supermercado 1 & & \\
Tienda 1 & $1,8 \times 10^{3}$ & $6,5 \times 10^{3}$ \\
Tienda 2 & $2,1 \times 10^{4}$ & $1,4 \times 10^{4}$ \\
Tienda 3 & $6,7 \times 10^{4}$ & $5,8 \times 10^{3}$ \\
Tienda 4 & $3,8 \times 10^{3}$ & $6,0 \times 10^{2}$ \\
Supermercado 2 & & \\
Tienda 5 & $3,8 \times 10^{4}$ & - \\
Tienda 6 & $1,9 \times 10^{3}$ & $1,3 \times 10^{3}$ \\
Supermercado 3 & & \\
Tienda 7 & $1,02 \times 10^{4}$ & $1,3 \times 10^{4}$ \\
Supermercado 4 & & \\
Tienda 8 & $2,2 \times 10^{4}$ & $8,5 \times 10^{4}$ \\
Supermercado 5 & & \\
Tienda 9 & $7,1 \times 10^{4}$ & $2,0 \times 10^{4}$ \\
Tienda 10 & $1,1 \times 10^{4}$ & $3,6 \times 10^{4}$ \\
\hline
\end{tabular}


resistencia. Los resultados del estudio se presentan en tablas, cuidando el anonimato de los supermercados y tiendas involucradas en el estudio.

\section{RESULTADOS}

Se aislaron 38 cepas de enterobacterias a partir de muestras de carne molida de recortes de res, en las 10

Tabla 2. Enterobacterias aisladas en carne molida comercializada en supermercados del distrito de Miraflores, Lima - Perú. Año 2016 (n=38).

\begin{tabular}{ll}
\hline Identificación & Nro. \\
\hline Serratia liquefaciens & 8 \\
Escherichia vulneris & 5 \\
Pantoea spp & 4 \\
Enterobacter cloacae & 3 \\
Klebsiella pneumoniae ssp ozaenae & 3 \\
Serratia fonticola & 3 \\
Serratia Orodifera & 2 \\
Citrobacter freundii & 1 \\
Salmonella pullorum & 1 \\
Raoultella ornithinolytica & 1 \\
Serratia marcescens & 1 \\
Yersinia enterocolitica & 1 \\
Citrobacter youngae & 1 \\
Cronobacter spp & 1 \\
Citrobacter braakii & 1 \\
Enterobacter amnigenus & 1 \\
Enterobacter aerogenes & 1 \\
\hline
\end{tabular}

tiendas de los 5 supermercados del distrito de Miraflores. La mediana del recuento bacteriano obtenido a partir de las muestras fue de $1,3 \times 10^{4} \mathrm{UFC} / \mathrm{g}$, el detalle se presenta en la tabla 1. Las especies de enterobacterias encontradas con mayor frecuencia fueron Serratia liquefaciens (21\%), Escherichia vulneris (13.16\%), Pantoea spp., (10.5\%). La descripción de todas las bacterias aisladas con sus respectivas frecuencias absolutas se presentan en la tabla 2 .

El 28,94\% (11/38) de cepas resultaron ser sospechosas de producir BLEE, siendo las especies encontradas principalmente: Enterobacter cloacae (2), C. freundii (1), E. vulneris (1), Pantoea spp. (1), S. fonticola (1), S. marcescens (1), Y. enterocolitica (1), $S$. liquefaciens, Cronobacter spp. (1) y C. braakii (1). Mientras, el 15.79\% (6/38) resultaron ser BLEE confirmados por medio de la prueba de doble disco en agar: E.cloacae (2), C. freundii (1), S. marcescens (1), $Y$. enterocolitica (1) y C. braakii (1). La descripción de las bacterias sospechosas y productoras de BLEE se presenta en la tabla 3 .

\section{DISCUSIÓN}

El presente estudio constituye el primero realizado en el país para determinar la presencia de especies de enterobacterias productoras de BLEE en muestras de carne molida de res comercializada en supermercados locales.

La ventaja de utilizar pruebas comerciales semiautomatizadas para la identificación bioquímica-

Tabla 3. Enterobacterias aisladas en la carne molida sospechosas y productoras de BLEE comercializada en supermercados del distrito de Miraflores, Lima - Perú. Año 2015.

\begin{tabular}{lll}
\hline Enterobacteria & Sospechoso* & BLEE** \\
\hline Enterobacter cloacae & 2 & 2 \\
Citrobacter freundii & 1 & 1 \\
Escherichia vulneris & 1 & - \\
Pantoea spp & 1 & - \\
Serratia fonticola & 1 & - \\
Serratia marcescens & 1 & 1 \\
Yersinia enterocolitica & 1 & 1 \\
Serratia liquefaciens & 1 & - \\
Cronobacter spp & 1 & - \\
Citrobacter braakii & 1 & 1 \\
Total & 11 & 6 \\
\hline
\end{tabular}

* Sospechoso: Metodología de difusión simple en agar

** BLEE: Metodología de doble disco (Cepa productora de $\beta$-lactamasas de espectro extendido) 
fenotípica bacteriana reside básicamente en que son pruebas estandarizadas y validadas, de fácil uso y bajo costo; además de que brindan identificaciones a partir de bases de datos de los patrones bioquímicos de las enterobacterias. Sin embargo, la subjetividad del color y el grado de parentesco entre especies puede resultar en falsos positivos; por lo que para mayor especificidad se recomiendan las pruebas moleculares (Castro et al., 2006). Así también, para el antibiograma se utilizaron discos con concentraciones comerciales estandarizadas previamente y establecidas por el CLSI, no se utilizó el método de Concentración Mínima Inhibitoria.

Las empresas industriales de alimentos deben asegurarse de que la carga microbiana cumpla con los criterios microbiológicos suscritos en la RM. No591- 2008 NTS-Nº71-MINSA/DIGESA-V.01. 6 ; 6.2; X.6 para carnes crudas, picadas y molidas, en donde se describen los límites permisibles para aerobios mesófilos, E. coli, Staphylococcus aureus, Clostridium perfringens, E. coli 0157:H7 y Salmonella spp. (ausencia/25 gramos)( Dirección General de Salud Ambiental [DIGESA], 2006). Si bien no se encontraron especies de enterobacterias indicadas en la normativa de DIGESA, sí se aisló Salmonella enterica serovar pullorum en una muestra de carne molida; la cual, según la norma, no debería estar presente. Adicionalmente, las demás especies de enterobacterias aisladas, incluyendo las BLEE confirmadas, no se encuentran especificadas en el criterio microbiológico de la NTS.

A pesar de ello, el recuento se realizó para poder tener un panorama global sobre la carga microbiana. La presencia de enterobacterias es importante ya que éstas podrían diseminar sus genes de resistencia a otros microorganismos asociados a enfermedades, los cuales podrían tener un potencial patógeno y cada vez comportarse más resistentes a antibióticos. Considerando que se ha descrito que las BLEE también ejercen resistencia cruzada frente a antibióticos como aminoglucósidos, tetraciclinas y cotrimoxazol (Perozo y Castellano, 2009).

Las especies de enterobacterias aisladas en este estudio no tienen mayor implicancia sanitaria; sin embargo pueden potencialmente generar indirectamente problemas debido a la capacidad de transferencia de su código genético a otros microorganismos (Philippon, Arlet y Lagrange, 1994). Adicionalmente, si bien la carne se consume en un punto de cocción (temperatura mínima interna de $71,1^{\circ} \mathrm{C}$ ) y las bacterias llegan a ser destruidas (United
States Department of Agriculture [USDA],2013), el ADN bacteriano no se desnaturalizarse ya que este proceso sucede a temperatura entre los $95-98{ }^{\circ} \mathrm{C}$ (Mullis, 1990). Por lo que podría inferirse que los genes de resistencia $\left(\mathrm{a} 71,1^{\circ} \mathrm{C}\right)$ no se degradarían y potencialmente serían transmisibles a bacterias saprofitas del hospedero.

Numerosos estudios mencionan como principal productor de BLEE a E. coli (Ojer-Usoz et al., 2013; Tekiner y Özpınar, 2016), agente que no se aisló en la presente investigación. Sin embargo, se determinó a otras enterobacterias productoras de BLEE como $E$. cloacae, $C$. freundii, $S$. marcescens, $Y$. enterocolitica y C. braakii.

Los hallazgos del género Serratia y Citrobacter son similares a lo reportado en el estudio de OjerUsoz et al., (2013). Después de E. coli $(74,1 \%)$, como segundo agente con mayor prevalencia está $S$. fonticola (13,9\%), seguido de C. koserii (2,8\%), y $E$. cloacae $(0,9 \%)$, todos identificados como BLEE con el método de difusión de doble disco en muestras de carne molida de res, pollo y cerdo de minimarkets en Navarra, España. Asimismo, Philippon et al., (1994) mencionan que los genes de resistencia mediados por plásmidos de una especie pueden transferirse a otras especies emparentadas. Se debe resaltar además que el recuento bacteriano descrito por Ojer et al., (2013) se realizó para las enterobacterias productoras de BLEE,agrupándose en cinco rangos de concentración (UFC/g); el 55,7\% de enterobacterias productoras de BLEE obtuvo una concentración menor a $10^{2} \mathrm{UFC} / \mathrm{g}$, el $30 \%$ entre $10^{2}$ y $10^{3} \mathrm{UFC} / \mathrm{g}$, mientras el $14,3 \%$ obtuvo concentraciones entre $10^{3}$ y $10^{4} \mathrm{UFC} / \mathrm{g}$; en donde las más altas concentraciones de enterobacterias en orden decreciente comprendieron a $S$. fonticola y E. coli. En nuestro estudio, también se determinó como enterobacterias productoras de BLEE al género Serratia; por otro lado, el recuento bacteriano se realizó en agar EMB, considerando enterobacterias productoras y no productoras de BLEE.

Geser, Stephan y Hächler (2012), determinaron la presencia de cepas productoras de BLEE en otro tipos de muestras como heces de bovinos sanos, leche de vacas con mastitis y en carne molida de res y cerdo, provenientes de una planta procesadora en Suiza. A diferencia de nuestro estudio, no encontraron cepas productoras de BLEE en carne molida.

Se han aislado microorganismos indicadores de contaminación y patógenos en instrumental de faena, 
como por ejemplo Proteus spp. (53,9\%), Citrobacter spp., (9\%) y Enterobacter spp., (1,2\%); así como Salmonella spp. resistente a cefalexina en carne molida. Lo que sugiere que como primer indicador de contaminación pueden encontrarse enterobacterias distintas a $E$. coli, hecho presente en nuestro estudio donde se encontró con mayor frecuencia el género Serratia. La presencia de Serratia spp. podría deberse a contaminación cruzada, ya que la bacteria se encuentra en agua potable, cañerías, botas, pediluvios, tracto gastrointestinal del hombre y animales, entre otros. Sin embargo, E coli, es el principal microorganismo indicador de contaminación fecal, su ausencia en nuestro estudio sugiere que la contaminación de la carne probablemente no se deba a residuos fecales sino por contaminación cruzada a través de otras fuentes (CODEX, 2005).

Según la NTS-N 071 , en 25 gramos debe estar ausente Salmonella spp. No obstante, se aisló Salmonella enterica serovar pullorum en una muestra de carne molida en una de las tiendas. Si bien es cierto $S$. pullorum está más adaptada a las aves que al hombre (Loureiro , Marques y Ramos, 2010), la contaminación por $S$. entérica serovar pullorum podría darse por contaminación cruzada (CODEX, 2005). Jensen et al., (2006), entre 2002 y 2004 aislaron también Salmonella no productora de BLEE en carne bovina molida en Dinamarca.

La presencia de $Y$. enterocolitica en la carne podría sugerir contaminación a partir de cerdos portadores (Borie et al., 1997) o en mataderos en donde también se benefician bovinos. Como panorama general, el biotipo $1 \mathrm{~A}$ de la bacteria es considerada no patógena para humanos y animales, no obstante, muchas de las cepas patógenas pertenecen a los biotipos 1B, 2, 3, 4 y 5 (Paixão et al., 2013). Sin embargo, en nuestro estudio se demostró que $Y$. enterocolitica puede ser productora de BLEE. Es necesario mencionar que no se caracterizó la patogenicidad de la bacteria. $Y$. enterocolitica además puede conllevar a potenciales daños sobre la salud, como diarreas agudas, ileítis terminal, ocasionando secuelas a largo plazo que siguen a la infección (Paixao et al., 2013).

\section{CONCLUSIONES}

El estudio reporta la presencia de un 15,7\% de enterobacterias productoras de $\beta$-lactamasas de espectro extendido en carne molida de recortes de res bovina, proveniente de supermercados de un distrito de Lima.
Las especies de enterobacterias aisladas más frecuentes fueron S. liquefaciens, E. vulneris y Pantoea spp. No se aisló E. coli como indicador de contaminación fecal.

\section{Correspondencia:}

Valeria Cortez Sandoval

Correo electrónico: valeria.cortez@upch.pe

\section{REFERENCIAS BIBLIOGRAFICAS}

1. Becton Dickinson GmbH. (2013). BD EMB Agar (Eosin Methylene Blue Agar), Modified. Instrucciones de uso. Heidelberg: Becton Dickinson GmbH. Recuperado de: https://www.bd.com/ resource. aspx?IDX=8765

2. Becton Dickinson GmbH. (2017). BD Mueller Hinton II Agar. BD Mueller Hinton II Agar $150 \mathrm{~mm}$. BD Mueller Hinton II Agar, Square. Instrucciones de uso. Heidelberg: Becton Dickinson GmbH. Recuperado de: https://www.bd.com/resource.aspx?IDX=8774

3. Biomerieux. (2017). Reference Guide API. 2018, de Biomerieux. Missouri: Biomerieux. Recuperado de: http://www.biomerieux-usa.com/sites/subsidiary_ us/files/18_api-ref-guide_v4.pdf

4. Blanc, V. (2007). Caracterización de cepas y de plásmidos de Enterobacteriaceae portadoras de betalactamasas de espectro extendido. Agosto, 2016, de Universidad Autónoma de Barcelona. Sitio web: http://www.tdx.cat/bitstream/handle/10803/ 3908/vbp1de1.pdf?sequence $=1$

5. Borie, C., Jara, M., Sanchez, M., San Martín, B., Arellano, C., Martinez, J., \& Prado, V. (1997). Aislamiento y Caracterización de Yersinia enterocolitica de Cerdos y Bovinos en Chile. J Vet Med B, 44,347-354.

6. Castro, S., Rodríguez, C., Perazzi, B., Radice, M., Sticotti, M., Muzio, H... Vay, C. (2006). Comparación de diferentes métodos para identificar las especies del género Proteus. Rev Argent Microbiol, 38(3), 119124.

7. Clinical Laboratory Standards Institute. (2015). Performance Standards for Antimicrobial Susceptibility Testing. Twenty-Fifth Informational Supplement. Wayne: Clinical Laboratory Standards Institute.

8. Codex Alimentarius. (2005). Código de prácticas de higiene para la carne (CAC/RCP 58/2005 Rev. 11-55). Agosto, 2016, de CODEX. Washington DC: Organización de las Naciones Unidas para la Agricultura y la Alimentación/Organización Mundial de la Salud.

9. Díaz, M., Hernández, J., Martínez-Martínez, L., Rodríguez-Baño, J., \& Pascual, A. (2009). 
Escherichia coli y Klebsiella pneumoniae productoras de betalactamasas de espectro extendido en hospitales españoles: segundo estudio multicéntrico (proyecto GEIH-BLEE 2006). Enferm Infecc Microbiol Clin, 27(9), 503-510.

10. Dirección General de Salud Ambiental. (2008). NTS N071 - MINSA/DIGESA-V.01. Norma sanitaria que establece los criterios microbiológicos de calidad sanitaria e inocuidad para alimentos y bebidas de consumo humano. Agosto, 2016, de MINSA Sitio web: http://www.digesa.sld.pe/norma_ consulta/RM\%20615-2003MINSA.pdf

11. García-Hernández A, García-Vázquez E, HernándezTorres A, Ruiz J, Yagüe G, Herrero J, Gómez J. (2011). Bacteriemias por Escherichia coli productor de betalactamasas de espectro extendido (BLEE): significación clínica y perspectivas actuales. Rev Esp. Quimioter, 24(2), pp.57-66.

12. Geser, N., Stephan, R., \& Hächler, H. (2012). Occurrence and characteristics of extended-spectrum $\beta$-lactamase (ESBL) producing Enterobacteriaceae in food producing animals, minced meat and raw milk. BMC Veterinary Research, 8(21),1-9.

13. Jarlier, V., Nicolas, M. H., Fournier, G., \& Phillippon, A. (1988). Extended-spectrum beta-lactamases conferring transferable resistance to newer betalactam agents in Enterobacteriaceae: hospital prevalence and susceptibility patterns. Rev Infect Dis, 10, 867-878.

11. Jay, J., Loessner, M., \& Golden, D. (2005). Modern Food Microbiology. (Seventh Edition) New York: Series Editor.

12. Jensen, L., Hasman, H., Agersø, Y., Emborg, H., \& Aarestrup, F. (2006). First description of an oxyiminocephalosporinresistant, ESBL-carrying Escherichia coli isolated from meat sold in Denmark. Journal of Antimicrobial Chemotherapy, 57(4), 79-794.

13. Loureiro, E., Marques, N., \& Ramos, F. (2018). Serotipos de Salmonella de origen humano identificados en el Estado de Pará (Brasil) entre 1991 y 2008. Rev Pan-Amaz Saude, 1(1),93-100.

14. Maturin, L., \& Peeler J. (2001). Aerobic Plate Count. En: BAM Council. Bacteriological Analytical Manual (BAM). Washington DC: USA Food and Drug Administration (FDA) Recuperado de: http:// www.fda.gov/Food/FoodScienceResearch/ LaboratoryMethods/ucm063346.htm

15. Mullis K. (1990). The unusual origin of the polymerase chain reaction. Scientific American, 262, 56-61.

16. Ojer-Usoz E, González D, Vitas A, Leiva J, GarcíaJalón I, Febles-Casquero A, Escolano M. (2013).
Prevalence of extended-spectrum $\beta$-lactamaseproducing Enterobacteriaceae in meat products sold in Navarra, Spain. Meat Science-Elsevier, 93, 316321.

17. Oliver A, Cantón A. (2005) Enterobacterias productoras de $\beta$-lactamasas plasmídicas de espectro extendido, 2016, de Servicios de Microbiología.

18. Madrid: Control Calidad SEIMC. Sitio web: http:// docplayer.es/13979417-Enterobacterias-productorasde-v-lactamasas-plasmidicas-de-espectro-extendido. html

19. Paixão, R., Moreno, L., De Gobbi, D., Raimundo, D., Hofer, E., Matté M... Moreno, A. (2013). Characterization of Yersinia enterocolitica Biotype 1A Strains Isolated from Swine Slaughterhouses and Markets. Hindawi Publishing Corporation Scientific World Journal, 2013, 769097 doi: $10.1155 / 2013 / 769097$

20. Perozo, A. \& Castellano, M. (2009). Detección de Betalactamasas de Espectro Extendido en cepas de la familia Enterobacteriaceae. Kasmera, 37(1), 25- 37.

21. Philippon, A., Arlet, G., \& Lagrange, P. H. (1994). Origin and impact of plasmid-mediated extendedspectrum beta-lactamases. European Journal of Clinical Microbiology and Infectious Diseases, 13(1), S17-S29.

22. Servicio Nacional de Seguridad Agraria. (2012). Decreto Supremo No015-2012-AG. Reglamento sanitario del faenado de animales de Abasto. Agosto, 2016, de MINAG. Lima: Servicio Nacional de Seguridad Agraria. Recuperado de: http:/www. senasa.gob.pe/senasa/wp-content/uploads/2014/10/ Decreto-Supremo-N\%C2\%BA-015-2012-AG.pdf

23. Seral, C., Pardos, M. \& Castillo, F. (2010). Betalactamasas de espectro extendido en enterobacterias distintas de Escherichia coli y Klebsiella. Enferm Infecc Microbiol Clin. 28 (1), 1218.

24. Tekiner, I. \& Özpınar H. (2016). Occurrence and characteristics of extended spectrum beta-lactamasesproducing Enterobacteriaceae from foods of animal origin. Brazilian Journey Of Microbiology, 47,444451.

25. United States Department of Agriculture. (2013). Carne Molida de Res e Inocuidad de Alimentos. Washington DC: United States Department of Agriculture Food Safety and Inspection Service. Recuperado de: https://www.fsis.usda.gov/wps/ portal/informational/en-espanol/hojasinformativas/ preparacion-de-las-carnes/enfoque-carne-molida-deres/carne-molida-res 